\title{
Mediterránea
}

\section{Editorial 2}

\section{Tendencias y demandas de investigación en infancia y comunicación //}

\section{Trends and Investigation requests on Children Communication}

La Asociación Infancia y Comunicación (Kids\&com) se ha propuesto la promoción de números monográficos en revistas científicas de prestigio que den cuenta de la tarea investigadora que muchos grupos de investigación están desarrollando. Con esa finalidad se publicó en 2014 un monográfico en la revista Comunicar coordinado por las Dras. García Galera y Valdivia (2014) sobre prosumidores mediáticos. En esa línea, ve ahora la luz en la Revista Mediterránea de Comunicación esta colección de investigaciones que hemos titulado "Infancia y Comunicación. Tendencias y demandas de investigación".

La selección de artículos publicados se ocupa de algunos de los más interesantes aspectos de la actual investigación sobre infancia y comunicación. Ciertamente quedan fuera algunos aspectos relevantes. No era posible ocuparse de todo. Pero no hay duda que los temas que finalmente publicamos recorren aspectos clave para la investigación actual en infancia y comunicación.

El Dr. Javier Gil, maestro e investigador en la UNED, propone uno de los temas de mayor potencial tanto para la investigación científica como para las profesiones comunicativas y educativas: el desarrollo y las posibilidades de las capacidades creadoras de los más jóvenes en un mundo digital.

La Dra. Leticia Porto, profesora de la Universidad Rey Juan Carlos, aborda un completo análisis de la más exitosa e innovadora producción audiovisual de 2015. Ciertamente se trata de un análisis de una propuesta de actualidad. Pero, además, coincido con los que sostienen que será una de las películas de la que seguiremos hablando durante mucho tiempo. La autora, especialista en el cine de animación de Pixar, aporta interesantes y rigurosas reflexiones.

Las Dras. Fabbro y Sánchez-Labella, de la Universidad Austral y de la Universidad de Sevilla respectivamente, mediante un análisis cualitativo de programas argentinos y españolas reflexionan sobre los valores de los contenidos audiovisuales en España y Argentina. 
La extensión de los productos audiovisuales de telerrealidad y sus contenidos son objeto de la investigación científica desde su aparición y consolidación en las parrillas. No podía faltar su presencia en nuestras propuestas de reflexión. En esta ocasión se trata de un artículo elaborado por Escudero y el Dr. Gabelas de la Universidad de Zaragoza.

El Dr. Santiago Gómez Amigo, profesor del CES Villanueva de la Universidad Complutense, con una dilatada experiencia en la investigación en programación, aborda la cuestión de los horarios infantiles. La comparación entre las franjas de protección y los horarios en los que los niños están ante las pantallas evidencia y desajuste entre la normativa y la realidad. Urge la revisión de las franjas para que la protección sea efectiva.

En cuanto a las tendencias en investigación publicitaria e infancia, el monográfico se ocupa del advergaming, probablemente uno de los hallazgos más llamativos de la comunicación persuasiva de los últimos años. El artículo lo firman las Dras. de la Universidad de Oviedo, Del Moral, Villalustre y Neira.

No puedo dejar de mencionar el excelente trabajo de la Dra.Victoria Tur, de la Universidad de Alicante, principal responsable de esta revista. Ha conseguido llevar a la Revista Mediterránea de Comunicación a muy reconocidas cotas de calidad. Su capacidad científica, su rigor y su continuado buen hacer han sido decisivos en toda la tarea. Desde aquí un particular agradecimiento.

Como se ve, la colección de artículos de este monográfico se ocupa de contenidos cinematograficos y televisivos, trata del papel de los más jóvenes como emisores y como receptores, lo hace con propuestas de investigación cualitativas y cuantitativas, etc. Es decir, estamos ante una valiosa referencia para conocer las líneas de investigación en infancia y comunicación actuales y futuras. Con ese propósito hicimos este trabajo. Confiamos en que quienes lo lean puedan aprovechar el esfuerzo realizado.

\title{
Bibliografla:
}

García, M.C. \& VAlDIVIA, A. (2014). Prosumidores mediáticos. Cultura participativa de las audiencias y responsabilidad de los medios [Media Prosumers. Participatory Culture of Audiences and Media Responsibility]. Comunicar, 43, 1013. http://dx.doi.org/10.3916/C43-2014-a2

Dr. José A. Ruiz San Román

\author{
Coordinador del dossier monográfico: Infancia y Comunicación. Tendencias y demandas de \\ investigación
}

Cómo citar: RUÍZ SAN ROMÁN, José A. (2016). Editorial: Tendencias y demandas de investigación en infancia y comunicación. Revista Mediterránea de Comunicación, 7(1), 7-8. Disponible en http://mediterranea-comunicacion.org/. DOI 10.14198/MEDCOM2016.7.1.14 